Research Article

\title{
Convergence of Infinite Family of Multivalued Quasi-Nonexpansive Mappings Using Multistep Iterative Processes
}

\author{
Renu Chugh and Sanjay Kumar \\ Department of Mathematics, Maharshi Dayanand University, Rohtak, India \\ Correspondence should be addressed to Sanjay Kumar; sanjay.sanju84@gmail.com
}

Received 5 August 2014; Accepted 27 September 2014; Published 24 December 2014

Academic Editor: Alicia Cordero

Copyright (C) 2014 R. Chugh and S. Kumar. This is an open access article distributed under the Creative Commons Attribution License, which permits unrestricted use, distribution, and reproduction in any medium, provided the original work is properly cited.

\begin{abstract}
We prove strong and weak convergence results using multistep iterative sequences for countable family of multivalued quasinonexpansive mappings by using some conditions in uniformly convex real Banach space. The results presented extended and improved the corresponding result of Zhang et al. (2013), Bunyawat and Suantai (2012), and some others from finite family, one countable family, and two countable families to $k$-number of countable families of multivalued quasi-nonexpansive mappings. Also we used a numerical example in $\mathrm{C}++$ computational programs to prove that the iterative scheme we used has better rate of convergence than other existing iterative schemes.
\end{abstract}

\section{Introduction}

Let $X$ be real Banach space. The convex subset $K$ is called proximal set if for each $x \in X$ there exists at least one $y \in K$ such that $\|x-y\|=d(x, K)=\inf \{\|x-k\|: k \in K\}$. Every closed convex subset of uniformly convex Banach spaces is proximal. We use the following notations for multivalued mappings:

$C(X)$ : collection of all nonempty compact subsets of $X$

$P(X)$ : collection of all nonempty proximal bounded subsets of $X$;

$C B(X)$ : collection of all nonempty bounded closed subsets of $X$.

Let $H$ be Hausdorff metric induced by $d$ of $K$ defined as $H(A, B)=\max \left\{\sup _{y \in B} d(x, B), \sup _{x \in A} d(A, y)\right\}$, for every $A, B \in C B(K)$. Let $T: K \rightarrow P(K)$; a multivalued mapping is said to be nonexpansive if $H(T x, T y) \leq\|x-y\|$, for all $x, y \in K$. An element $p \in K$ is called fixed point of $T$ if $p \in T(p)$, where the set of all fixed points of $T$ is denoted by $F_{T}$. The mapping $T$ is said to be quasi nonexpansive if
$F_{T} \neq \varnothing$ and $H(T x, T p) \leq\|x-p\|$ for all $x \in K$ and $p \in F_{T}$. It is known that every nonexpansive multivalued mapping with $F_{T} \neq \varnothing$ is quasi nonexpansive, but there exist quasi-nonexpansive mappings which are not nonexpansive. It is well known that if $T$ is quasi-nonexpansive mapping, then $F_{T}$ is closed.

Definition 1. A map $T: K \rightarrow C B(K)$ is called hemicompact if, for any sequence $\left\{x_{n}\right\}$ in $K$ such that $d\left(x_{n}, T x_{n}\right) \rightarrow 0$ as $n \rightarrow \infty$, there exists a subsequence $\left\{x_{n_{k}}\right\}$ of $\left\{x_{n}\right\}$ such that $x_{n_{k}} \rightarrow p \in K$. It is clear that if $K$ is compact, then every multivalued mapping $T$ is hemicompact.

Definition 2. A Banach space $X$ is said to satisfy Opial's condition if $z \neq y$ imply that

$$
\lim _{n \rightarrow \infty} \sup \left\|x_{n}-z\right\|<\lim _{n \rightarrow \infty} \sup \left\|x_{n}-y\right\| .
$$

The study of multivalued nonexpansive mappings is harder than the corresponding theory of single-valued nonexpansive mappings. In 1969, Nadler Jr. [1] proved the convergence theorem for multivalued contraction mappings. Then in 1973, Markin [2] studied the multivalued contraction and nonexpansive mappings in Hausdorff metric space. Later in 
1997, Hu et al. [3] proved the convergence theorems for finding common fixed point of two multivalued nonexpansive mappings that satisfies certain contractive conditions. Sastry and Babu [4] proved the convergence of Mann and Ishikawa iterates to a fixed point $q$ of the multivalued mapping $T$ with fixed point $p$ under certain conditions. They proved with the help of example that limit of the sequence is different from the point of initial choice. Then Abbas et al. [5] introduced the new one-step iterative processes to compute the common fixed point of two multivalued nonexpansive mappings in a real uniformly convex Banach space. Let $S, T: K \rightarrow P(K)$ be two multivalued nonexpansive mappings. They introduced iteration as follows:

$$
\begin{gathered}
x_{0} \in K, \\
x_{n+1}=a_{n} x_{n}+b_{n} y_{n}+c_{n} z_{n}, \quad n \in N,
\end{gathered}
$$

where $y_{n} \in T x_{n}, z_{n} \in S x_{n}$, such that $\left\|y_{n}-p\right\| \leq d\left(p, S x_{n}\right)$ and $\left\|z_{n}-p\right\| \leq d\left(p, T x_{n}\right)$ for $p \in F(S) \cap F(T)$ and $a_{n}, b_{n}, c_{n} \in$ $(0,1)$ satisfying $a_{n}+b_{n}+c_{n} \leq 1$. Then they obtained strong convergence theorems for the proposed process under some basic boundary conditions.

In 2012 Bunyawat and Suantai [6] introduced the one-step iterative process as follows:

$$
x_{n+1}=\alpha_{n, 0} x_{n}+\sum_{i=1}^{\infty} \alpha_{n, i} x_{n, i}
$$

where the sequence $\left\{\alpha_{n, i}\right\}_{i=0}^{\infty} \subset[0,1)$ satisfying $\sum_{i=0}^{\infty} \alpha_{n, i}=$ 1 and $x_{n, i} \in T_{i} x_{n}$ such that $d\left(p, x_{n, i}\right)=d\left(p, T_{i} x_{n}\right)$ for $i \in N$. They proved the convergence of iterative processes to common fixed point of countable family of multivalued quasi-nonexpansive mappings in uniformly convex Banach space.

Then Zhang et al. in 2013 introduced the two-step iterative process as follows:

$$
\begin{array}{r}
x_{n+1}=\alpha_{n, 0} x_{n}+\sum_{i=1}^{\infty} \alpha_{n, i} y_{n, i}, \\
y_{n}=\beta_{n, 0} x_{n}+\sum_{i=1}^{\infty} \beta_{n, i} x_{n, i},
\end{array}
$$

where the sequences $\left\{\alpha_{n, i}\right\}_{i=0}^{\infty},\left\{\beta_{n, i}\right\}_{i=0}^{\infty} \subset[0,1)$ satisfying $\sum_{i=0}^{\infty} \alpha_{n, i}=1, \sum_{i=0}^{\infty} \beta_{n, i}=1, y_{n, i} \in T_{i} y_{n}$ such that $d\left(p, y_{n, i}\right)=$ $d\left(p, T_{i} y_{n}\right)$ and $x_{n, i} \in T_{i} x_{n}$ such that $d\left(p, x_{n, i}\right)=d\left(p, T_{i} x_{n}\right)$ for $i \in N$. Zhang et al. extended the results of Bunyawat and Suantai from one countable family to two countable families and also gave a new proof for the iteration used in the paper of Abbas et al. [5].
In the same year Ahmed and Altwqi introduced the threestep iterative process as follows:

$$
\begin{array}{r}
x_{n+1}=\alpha_{n, 0} x_{n}+\sum_{i=1}^{p} \alpha_{n, i} y_{n, i}, \\
y_{n}=\beta_{n, 0} x_{n}+\sum_{i=1}^{p} \beta_{n, i} z_{n, i}, \\
z_{n}=\gamma_{n, 0} x_{n}+\sum_{i=1}^{p} \gamma_{n, i} x_{n, i},
\end{array}
$$

where $z_{n, i} \in T_{i} z_{n}, y_{n, i} \in T_{i} y_{n}$, and $x_{n, i} \in T_{i} x_{n}$ and the sequences $\left\{\alpha_{n, i}\right\}_{i=0}^{p},\left\{\beta_{n, i}\right\}_{i=0}^{p},\left\{\gamma_{n, i}\right\}_{i=0}^{p} \subset[0,1)$ satisfying $\sum_{i=0}^{p} \alpha_{n, i}=1 \sum_{i=0}^{p} \beta_{n, i}=1, \sum_{i=0}^{p} \gamma_{n, i}=1$, and proved the strong and weak convergence results for three finite families of multivalued nonexpansive mappings.

Different iterative processes have been used to approximate fixed points of multivalued mappings. Many authors have intensively studied the fixed point theorems and got some results. At the same time, they extended these results to many discipline branches, such as control theory, convex optimization, variational inequalities, differential inclusion, and economics (see [7-19]).

Motivated by $[6,20-22]$, in this paper, we extended the result of Zhang et al. [21] from two countable families to $k$ number of countable families and proved weak and strong convergence results of two new multistep iterative processes to common fixed point of countable family of multivalued quasi-nonexpansive mappings in a uniformly convex Banach space. Also with the help of numerical example we compare the convergence step of two different multistep iterative processes. We use the following iteration processes:

$$
\begin{aligned}
x_{n+1} & =\alpha_{n, 0} x_{n}+\sum_{i=1}^{\infty} \alpha_{n, i} u_{n, i}^{1}, \\
y_{n}^{j} & =\beta_{n, 0}^{j} x_{n}+\sum_{i=1}^{\infty} \beta_{n, i}^{j} u_{n, i}^{j+1}, \quad j=1,2, \ldots, k-2, \\
y_{n}^{k-1} & =\beta_{n, 0}^{k-1} x_{n}+\sum_{i=1}^{\infty} \beta_{n, i}^{k-1} u_{n, i}^{k}, \\
x_{n+1} & =\alpha_{n, 0} y_{n}^{1}+\sum_{i=1}^{\infty} \alpha_{n, i} u_{n, i}^{1}, \\
y_{n}^{j} & =\beta_{n, 0}^{j} y_{n}^{j+1}+\sum_{i=1}^{\infty} \beta_{n, i}^{j} u_{n, i}^{j+1}, \quad j=1,2, \ldots, k-2, \\
y_{n}^{k-1} & =\beta_{n, 0}^{k-1} x_{n}+\sum_{i=1}^{\infty} \beta_{n, i}^{k-1} u_{n, i}^{k},
\end{aligned}
$$

where $u_{n, i}^{j} \in T_{i}^{j} y_{n}^{j}, j=1,2, \ldots, k-1$ such that $d\left(p, u_{n, i}^{j}\right)=$ $d\left(p, T_{i}^{j} y_{n}^{j}\right), j=1,2, \ldots, k-1$ and $u_{n, i}^{k} \in T_{i}^{k} x_{n}$ such that $d\left(p, u_{n, i}^{k}\right)=d\left(p, T_{i}^{k} x_{n}\right),\left\{\alpha_{n, i}\right\}_{i=0}^{\infty}$, and $\left\{\beta_{n, i}^{j}\right\}_{i=0}^{\infty}, j=1,2, \ldots, k-$ 1 are sequences in $[0,1]$ which satisfies $\sum_{i=0}^{\infty} \alpha_{n, i}=1$ and 
$\sum_{i=0}^{\infty} \beta_{n, i}^{j}=1, j=1,2, \ldots, k-1, \limsup _{n \rightarrow \infty} \alpha_{n, 0}<$ 1 and $\lim \sup _{n \rightarrow \infty} \beta_{n, 0}^{j}<1, j=1,2, \ldots, k-2$, and $\liminf _{n \rightarrow \infty} \alpha_{n, 0} \alpha_{n, m}>0$ and $\liminf _{n \rightarrow \infty} \beta_{n, 0}^{j} \beta_{n, m}^{j}>0, j=$ $1,2, \ldots, k-1$.

Remark 3. If $k=1$ and 2, then multistep iteration (6) reduces to one-step and two-step iterations (3) and (4) defined by Bunyawat and Suantai and Zhang et al. whereas for $k=3$, $i=p$ multistep iteration (6) reduces to finite three-step iteration (5) defined by Ahmed and Altwqi.

Lemma 4 (see [6]). Let $X$ be a uniformly convex Banach space, $r>0$ a positive number, and $B_{r}(0)$ a closed ball of $X$. Then, for any given sequence $\left\{x_{n}\right\}_{n=1}^{\infty} \subset B_{r}(0)$ and for any given sequence $\left\{\lambda_{i}\right\}_{i=1}^{\infty}$ of positive number with $\sum_{n=1}^{\infty} \lambda_{n}=1$, there exists a continuous, strictly increasing, and convex function $g:[0,2 r) \rightarrow[0, \infty)$ with $g(0)=0$ such that, for any positive integer $i, j$ with $i \neq j$

$$
\left\|\sum_{n=1}^{\infty} \lambda_{n} x_{n}\right\|^{2} \leq \sum_{n=1}^{\infty} \lambda_{n}\left\|x_{n}\right\|^{2}-\lambda_{i} \lambda_{j} g\left(\left\|x_{i}-x_{j}\right\|\right) .
$$

Lemma 5 (see [23]). Suppose that $X$ is a uniformly convex Banach space and $0<p \leq t_{n} \leq q<1$ for all positive integers n. Also suppose that $\left\{x_{n}\right\}$ and $\left\{y_{n}\right\}$ are two sequences of $E$ such that $\lim _{\sup _{n \rightarrow \infty}}\left\|x_{n}\right\| \leq r$, $\lim _{\sup _{n \rightarrow \infty}}\left\|y_{n}\right\| \leq r$, and $\lim _{n \rightarrow \infty}\left\|t x_{n}+(1-t) y_{n}\right\|=r$ hold for some $r \geq 0$; then $\lim _{n \rightarrow \infty}\left\|x_{n}-y_{n}\right\|=0$.

Lemma 6 (see $[24,25])$. Let $\left\{a_{n}\right\}$ be a sequence of nonnegative real numbers satisfying the following property: $a_{n+1} \leq(1-$ $\left.t_{n}\right) a_{n}+b_{n}+t_{n} c_{n}$, where $\left\{t_{n}\right\},\left\{b_{n}\right\}$, and $\left\{c_{n}\right\}$ satisfy the following restrictions:

(i) $\sum_{n=1}^{\infty} t_{n}=\infty$;

(ii) $\sum_{n=1}^{\infty} b_{n}<\infty$;

(iii) $\lim \sup _{n \rightarrow \infty} c_{n} \leq 0$

Then, $\left\{a_{n}\right\}$ converges to zero as $n \rightarrow \infty$.

\section{Main Results}

2.1. Weak and Strong Convergence Results for New Multistep Iterative Scheme (6)

Theorem 7. Let $K$ be a nonempty closed convex subset of a uniformly convex Banach space $X$ with Opial's condition. For $i \in N$, let $\left\{T_{i}^{j}\right\}_{j=1}^{k}$ be $k$ sequences of multivalued quasi-nonexpansive mappings from $K$ into $P(K)$ with $F=$ $\cap_{j=1}^{k}\left[\cap_{i=1}^{\infty} T_{i}^{j}\right] \neq \varnothing$ and $p \in F$. Let $\left\{x_{n}\right\}$ be the sequence defined by (6) and then it converges weakly to a point $q \in F$.
Proof. Let $p \in F$; first we prove that $\left\{x_{n}\right\}$ is bounded and $\lim _{n \rightarrow \infty}\left\|x_{n}-p\right\|$ exists. Now from Lemma 5 and (6), we have

$$
\begin{aligned}
\left\|y_{n}^{k-1}-p\right\|^{2}= & \left\|\beta_{n, 0}^{k-1} x_{n}+\sum_{i=1}^{\infty} \beta_{n, i}^{k-1} u_{n, i}^{k}-p\right\|^{2} \\
\leq & \beta_{n, 0}^{k-1}\left\|x_{n}-p\right\|^{2}+\sum_{i=1}^{\infty} \beta_{n, i}^{k-1}\left\|u_{n, i}^{k}-p\right\|^{2} \\
& -\beta_{n, 0}^{k-1} \beta_{n, m}^{k-1} g\left(\left\|x_{n}-u_{n, m}^{k}\right\|\right) \\
\leq & \beta_{n, 0}^{k-1}\left\|x_{n}-p\right\|^{2}+\sum_{i=1}^{\infty} \beta_{n, i}^{k-1}\left(d\left(u_{n, i}^{k}, T_{i}^{k} p\right)\right)^{2} \\
& -\beta_{n, 0}^{k-1} \beta_{n, m}^{k-1} g\left(\left\|x_{n}-u_{n, m}^{k}\right\|\right) \\
\leq & \beta_{n, 0}^{k-1}\left\|x_{n}-p\right\|^{2}+\sum_{i=1}^{\infty} \beta_{n, i}^{k-1}\left(H\left(T_{i}^{k} x_{n}, T_{i}^{k} p\right)\right)^{2} \\
& -\beta_{n, 0}^{k-1} \beta_{n, m}^{k-1} g\left(\left\|x_{n}-u_{n, m}^{k}\right\|\right) \\
\leq & \beta_{n, 0}^{k-1}\left\|x_{n}-p\right\|^{2}+\sum_{i=1}^{\infty} \beta_{n, i}^{k-1}\left\|x_{n}-p\right\|^{2} \\
& -\beta_{n, 0}^{k-1} \beta_{n, m}^{k-1} g\left(\left\|x_{n}-u_{n, m}^{k}\right\|\right) \\
\leq & \left\|x_{n}-p\right\|^{2}-\beta_{n, 0}^{k-1} \beta_{n, m}^{k-1} g\left(\left\|x_{n}-u_{n, m}^{k}\right\|\right) .
\end{aligned}
$$

From (6), we get

$$
\begin{aligned}
\| y_{n}^{k-2}- & p \|^{2} \\
= & \left\|\beta_{n, 0}^{k-2} x_{n}+\sum_{i=1}^{\infty} \beta_{n, i}^{k-2} u_{n, i}^{k-1}-p\right\|^{2} \\
\leq & \beta_{n, 0}^{k-2}\left\|x_{n}-p\right\|^{2}+\sum_{i=1}^{\infty} \beta_{n, i}^{k-2}\left\|u_{n, i}^{k-1}-p\right\|^{2} \\
& -\beta_{n, 0}^{k-2} \beta_{n, m}^{k-2} g\left(\left\|x_{n}-u_{n, m}^{k-1}\right\|\right) \\
\leq & \beta_{n, 0}^{k-2}\left\|x_{n}-p\right\|^{2}+\sum_{i=1}^{\infty} \beta_{n, i}^{k-2}\left(d\left(u_{n, i}^{k-1}, T_{i}^{k-1} p\right)\right)^{2} \\
& -\beta_{n, 0}^{k-2} \beta_{n, m}^{k-2} g\left(\left\|x_{n}-u_{n, m}^{k-1}\right\|\right) \\
\leq & \beta_{n, 0}^{k-2}\left\|x_{n}-p\right\|^{2}+\sum_{i=1}^{\infty} \beta_{n, i}^{k-2}\left(H\left(T_{i}^{k-1} y_{n}^{k-1}, T_{i}^{k-1} p\right)\right)^{2} \\
& -\beta_{n, 0}^{k-2} \beta_{n, m}^{k-2} g\left(\left\|x_{n}-u_{n, m}^{k-1}\right\|\right) \\
\leq & \beta_{n, 0}^{k-2}\left\|x_{n}-p\right\|^{2}+\sum_{i=1}^{\infty} \beta_{n, i}^{k-2}\left\|y_{n}^{k-1}-p\right\|^{2} \\
& -\beta_{n, 0}^{k-2} \beta_{n, m}^{k-2} g\left(\left\|x_{n}-u_{n, m}^{k-1}\right\|\right)
\end{aligned}
$$




$$
\begin{aligned}
\leq & \beta_{n, 0}^{k-2}\left\|x_{n}-p\right\|^{2} \\
& +\sum_{i=1}^{\infty} \beta_{n, i}^{k-2}\left[\left\|x_{n}-p\right\|^{2}-\beta_{n, 0}^{k-1} \beta_{n, m}^{k-1} g\left(\left\|x_{n}-u_{n, m}^{k}\right\|\right)\right] \\
& -\beta_{n, 0}^{k-2} \beta_{n, m}^{k-2} g\left(\left\|x_{n}-u_{n, m}^{k-1}\right\|\right) \\
\leq & \left\|x_{n}-p\right\|^{2}-\left(1-\beta_{n, 0}^{k-2}\right) \beta_{n, 0}^{k-1} \beta_{n, m}^{k-1} g\left(\left\|x_{n}-u_{n, m}^{k}\right\|\right) .
\end{aligned}
$$

Similarly, we have

$$
\begin{aligned}
& \left\|y_{n}^{k-3}-p\right\|^{2} \\
& =\left\|\beta_{n, 0}^{k-3} x_{n}+\sum_{i=1}^{\infty} \beta_{n, i}^{k-3} u_{n, i}^{k-2}-p\right\|^{2} \\
& \leq \beta_{n, 0}^{k-3}\left\|x_{n}-p\right\|^{2}+\sum_{i=1}^{\infty} \beta_{n, i}^{k-3}\left\|u_{n, i}^{k-2}-p\right\|^{2} \\
& -\beta_{n, 0}^{k-3} \beta_{n, m}^{k-3} g\left(\left\|x_{n}-u_{n, m}^{k-2}\right\|\right) \\
& \leq \beta_{n, 0}^{k-3}\left\|x_{n}-p\right\|^{2}+\sum_{i=1}^{\infty} \beta_{n, i}^{k-3}\left(d\left(u_{n, i}^{k-2}, T_{i}^{k-2} p\right)\right)^{2} \\
& -\beta_{n, 0}^{k-3} \beta_{n, m}^{k-3} g\left(\left\|x_{n}-u_{n, m}^{k-2}\right\|\right) \\
& \leq \beta_{n, 0}^{k-3}\left\|x_{n}-p\right\|^{2} \\
& +\sum_{i=1}^{\infty} \beta_{n, i}^{k-3}\left(H\left(T_{i}^{k-2} y_{n}^{k-2}, T_{i}^{k-2} p\right)\right)^{2} \\
& -\beta_{n, 0}^{k-3} \beta_{n, m}^{k-3} g\left(\left\|x_{n}-u_{n, m}^{k-2}\right\|\right) \\
& \leq \beta_{n, 0}^{k-3}\left\|x_{n}-p\right\|^{2}+\sum_{i=1}^{\infty} \beta_{n, i}^{k-3}\left\|y_{n}^{k-2}-p\right\|^{2} \\
& -\beta_{n, 0}^{k-3} \beta_{n, m}^{k-3} g\left(\left\|x_{n}-u_{n, m}^{k-2}\right\|\right) \\
& \left\|y_{n}^{k-3}-p\right\|^{2} \\
& \leq \beta_{n, 0}^{k-3}\left\|x_{n}-p\right\|^{2} \\
& +\sum_{i=1}^{\infty} \beta_{n, i}^{k-3}\left[\left\|x_{n}-p\right\|^{2}\right. \\
& \left.-\left(1-\beta_{n, 0}^{k-2}\right) \beta_{n, 0}^{k-1} \beta_{n, m}^{k-1} g\left(\left\|x_{n}-u_{n, m}^{k}\right\|\right)\right] \\
& -\beta_{n, 0}^{k-3} \beta_{n, m}^{k-3} g\left(\left\|x_{n}-u_{n, m}^{k-2}\right\|\right) \\
& \leq\left\|x_{n}-p\right\|^{2} \\
& -\left(1-\beta_{n, 0}^{k-3}\right)\left(1-\beta_{n, 0}^{k-2}\right) \beta_{n, 0}^{k-1} \beta_{n, m}^{k-1} g\left(\left\|x_{n}-u_{n, m}^{k}\right\|\right) .
\end{aligned}
$$

Now continuing like this at last, we get

$$
\begin{aligned}
\left\|y_{n}^{1}-p\right\|^{2} \leq & \left\|x_{n}-p\right\|^{2} \\
& -\left(1-\beta_{n, 0}^{1}\right) \cdots\left(1-\beta_{n, 0}^{k-3}\right) \\
& \times\left(1-\beta_{n, 0}^{k-2}\right) \beta_{n, 0}^{k-1} \beta_{n, m}^{k-1} g\left(\left\|x_{n}-u_{n, m}^{k}\right\|\right) \\
\left\|x_{n+1}-p\right\|^{2}= & \left\|\alpha_{n, 0} x_{n}+\sum_{i=1}^{\infty} \alpha_{n, i} u_{n, i}^{1}-p\right\|^{2} \\
\leq & \alpha_{n, 0}\left\|x_{n}-p\right\|^{2}+\sum_{i=1}^{\infty} \alpha_{n, i}\left\|u_{n, i}^{1}-p\right\|^{2} \\
\leq & \alpha_{n, 0}\left\|x_{n}-p\right\|^{2}+\sum_{i=1}^{\infty} \alpha_{n, i}\left(d\left(u_{n, i}^{1}, T_{i}^{1} p\right)\right)^{2} \\
\leq & \alpha_{n, 0}\left\|x_{n}-p\right\|^{2}+\sum_{i=1}^{\infty} \alpha_{n, i}\left(H\left(T_{i}^{1} y_{n}^{1}, T_{i}^{1} p\right)\right)^{2} \\
\leq & \alpha_{n, 0}\left\|x_{n}-p\right\|^{2}+\sum_{i=1}^{\infty} \alpha_{n, i}\left\|y_{n}^{1}-p\right\|^{2} .
\end{aligned}
$$

By putting (13) in (14), we get

$$
\begin{aligned}
\left\|x_{n+1}-p\right\|^{2} \leq & \left\|x_{n}-p\right\|^{2} \\
& -\left(1-\alpha_{n, 0}\right)\left(1-\beta_{n, 0}^{1}\right) \cdots\left(1-\beta_{n, 0}^{k-3}\right) \\
& \times\left(1-\beta_{n, 0}^{k-2}\right) \beta_{n, 0}^{k-1} \beta_{n, m}^{k-1} g\left(\left\|x_{n}-u_{n, m}^{k}\right\|\right) .
\end{aligned}
$$

So from (15), we say that $\left\{\left\|x_{n}-p\right\|\right\}$ is nondecreasing and bounded and hence, $\left\{x_{n}\right\}$ is bounded and $\lim _{n \rightarrow \infty}\left\|x_{n}-p\right\|$ exists.

Now we prove that $\lim _{n \rightarrow \infty}\left\|x_{n}-u_{n, m}^{k}\right\|=0$ and $\lim _{n \rightarrow \infty}\left\|y_{n}^{j}-u_{n, m}^{j}\right\|=0$, where $j=1,2, \ldots, k-1$.

From (15), we can write as

$$
\begin{aligned}
& \left(1-\alpha_{n, 0}\right)\left(1-\beta_{n, 0}^{1}\right) \cdots\left(1-\beta_{n, 0}^{k-3}\right) \\
& \quad \times\left(1-\beta_{n, 0}^{k-2}\right) \beta_{n, 0}^{k-1} \beta_{n, m}^{k-1} g\left(\left\|x_{n}-u_{n, m}^{k}\right\|\right) \\
& \leq\left\|x_{n}-p\right\|^{2}-\left\|x_{n+1}-p\right\|^{2} .
\end{aligned}
$$

Since we assume that $\limsup _{n \rightarrow \infty} \alpha_{n, 0}<1, \lim _{\sup _{n \rightarrow \infty}} \beta_{n, 0}^{j}<$ $1, j=1,2, \ldots, k-2, \liminf _{n \rightarrow \infty} \alpha_{n, 0} \alpha_{n, m}>0$, $\liminf _{n \rightarrow \infty} \beta_{n, 0}^{j} \beta_{n, i}^{j}>0, j=1,2, \ldots, k-1$, and $\lim _{n \rightarrow \infty} \| x_{n}-$ $p \|$ exist, we have $\lim _{n \rightarrow \infty} g\left(\left\|x_{n}-u_{n, m}^{k}\right\|\right)=0$ and from the continuity of $g$, we have

$$
\lim _{n \rightarrow \infty}\left\|x_{n}-u_{n, m}^{k}\right\|=0, \quad \text { for each } m \in N .
$$

Now we will prove that $\lim _{n \rightarrow \infty}\left\|x_{n}-u_{n, m}^{j}\right\|=0$, for each $j=1,2, \ldots, k-1$.

From (9) and (10), we have

$$
\left\|y_{n}^{k-2}-p\right\|^{2} \leq\left\|x_{n}-p\right\|^{2}-\beta_{n, 0}^{k-2} \beta_{n, m}^{k-2} g\left(\left\|x_{n}-u_{n, m}^{k-1}\right\|\right) .
$$


Now, by putting (10) in (12), we have

$$
\begin{aligned}
\left\|y_{n}^{k-3}-p\right\|^{2} \leq & \left\|x_{n}-p\right\|^{2} \\
& -\left(1-\beta_{n, 0}^{k-3}\right) \beta_{n, 0}^{k-2} \beta_{n, m}^{k-2} g\left(\left\|x_{n}-u_{n, m}^{k-1}\right\|\right) .
\end{aligned}
$$

Now continuing like this at last, we have

$$
\begin{aligned}
\left\|x_{n+1}-p\right\|^{2} \leq & \left\|x_{n}-p\right\|^{2} \\
& -\left(1-\alpha_{n, 0}\right)\left(1-\beta_{n, 0}^{1}\right) \cdots\left(1-\beta_{n, 0}^{k-3}\right) \\
& \times \beta_{n, 0}^{k-2} \beta_{n, k}^{k-2} g\left(\left\|x_{n}-u_{n, m}^{k-1}\right\|\right) ;
\end{aligned}
$$

we can write it as

$$
\begin{aligned}
& \left(1-\alpha_{n, 0}\right)\left(1-\beta_{n, 0}^{1}\right) \cdots\left(1-\beta_{n, 0}^{k-3}\right) \beta_{n, 0}^{k-2} \beta_{n, k}^{k-2} g\left(\left\|x_{n}-u_{n, m}^{k-1}\right\|\right) \\
& \leq\left\|x_{n}-p\right\|^{2}-\left\|x_{n+1}-p\right\|^{2} .
\end{aligned}
$$

Since we assume that $\lim _{\sup } \rightarrow \infty \alpha_{n, 0}<1, \lim \sup _{n \rightarrow \infty} \beta_{n, 0}^{j}<$ $1, j=1,2, \ldots, k-2, \liminf _{n \rightarrow \infty} \alpha_{n, 0} \alpha_{n, m}>0$, $\liminf _{n \rightarrow \infty} \beta_{n, 0}^{j} \beta_{n, i}^{j}>0, j=1,2, \ldots, k-1$, and $\lim _{n \rightarrow \infty} \| x_{n}-$ $p \|$ exists, we have $\lim _{n \rightarrow \infty} g\left(\left\|x_{n}-u_{n, m}^{k-1}\right\|\right)=0$ and from the continuity of $g$, we have

$$
\lim _{n \rightarrow \infty}\left\|x_{n}-u_{n, m}^{k-1}\right\|=0, \quad \text { for each } m \in N
$$

So by repeating these steps for different values of $j=$ $1,2, \ldots, k-2$, we have

$$
\begin{array}{r}
\lim _{n \rightarrow \infty}\left\|x_{n}-u_{n, m}^{j}\right\|=0, \quad \text { for each } m \in N, \\
\text { where } j=1,2, \ldots, k-1 .
\end{array}
$$

Next, we prove that $\lim _{n \rightarrow \infty}\left\|y_{n}^{j}-u_{n, m}^{j}\right\|=0$, for each $m \in N$, where $j=1,2, \ldots, k-1$.

From (6), we have

$$
\begin{aligned}
\left\|x_{n}-y_{n}^{j}\right\| & =\left\|\beta_{n, 0}^{j} x_{n}+\sum_{i=1}^{\infty} \beta_{n, i}^{j} u_{n, i}^{j+1}-x_{n}\right\| \\
& \leq \sum_{i=1}^{\infty} \beta_{n, i}^{j}\left\|x_{n}-u_{n, i}^{j+1}\right\|
\end{aligned}
$$

where $j=1,2, \ldots, k-2$.

From (23), we have

$$
\lim _{n \rightarrow \infty}\left\|x_{n}-y_{n}^{j}\right\|=0, \quad \text { where } j=1,2, \ldots, k-1 .
$$

By using triangle inequality, we have

$$
\left\|y_{n}^{j}-u_{n, m}^{j}\right\| \leq\left\|y_{n}^{j}-x_{n}\right\|+\left\|x_{n}-u_{n, m}^{j}\right\| .
$$

Together with (23) and (25), we have

$$
\lim _{n \rightarrow \infty}\left\|y_{n}^{j}-u_{n, m}^{j}\right\|=0, \quad \text { where } j=1,2, \ldots, k-1,
$$

for each $m \in N$.
Now, we prove that $\left\{x_{n}\right\}$ converges weakly to a point $q \in F$. Since we have proved that $\left\{x_{n}\right\}$ is bounded, there exists a subsequence $\left\{x_{n_{k}}\right\}_{k=1}^{\infty}$ of $\left\{x_{n}\right\}$ such that $x_{n_{k}}$ converges weakly to $q \in K$; using (25), we can say that $y_{n_{k}}^{j}$ converges weakly to $q \in K$, for $j=1,2, \ldots, k-1$. Now suppose that there exist $i \in N$, such that $T_{i}^{j} q \neq q$, for $j=1,2, \ldots, k$; then by Opial's condition we have

$$
\begin{array}{r}
\limsup _{n \rightarrow \infty}\left\|x_{n_{k}}-q\right\|<\limsup _{n \rightarrow \infty}\left\|x_{n_{k}}-T_{i}^{k} q\right\| \\
\limsup _{n \rightarrow \infty}\left\|y_{n_{k}}^{j}-q\right\|<\limsup _{n \rightarrow \infty}\left\|y_{n_{k}}^{j}-T_{i}^{j} q\right\|, \\
\text { where } j=1,2, \ldots, k-1 .
\end{array}
$$

As $\left\{T_{i}^{j}\right\}_{j=1}^{k}$ are $k$-multivalued quasi-nonexpansive mappings, we have

$$
\begin{aligned}
\left\|x_{n_{k}}-T_{i}^{k} q\right\| & \leq\left\|x_{n_{k}}-u_{n_{k}, i}^{k}\right\|+\left\|u_{n_{k}, i}^{k}-T_{i}^{k} q\right\| \\
& \leq\left\|x_{n_{k}}-u_{n_{k}, i}^{k}\right\|+d\left(T_{i}^{k} x_{n_{k}}, T_{i}^{k} q\right) \\
& \leq\left\|x_{n_{k}}-u_{n_{k}, i}^{k}\right\|+H\left(T_{i}^{k} x_{n_{k}}, T_{i}^{k} q\right) \\
& \leq\left\|x_{n_{k}}-u_{n_{k}, i}^{k}\right\|+\left\|x_{n_{k}}-q\right\|, \\
\left\|y_{n_{k}}^{j}-T_{i}^{j} q\right\| & \leq\left\|y_{n_{k}}^{j}-u_{n_{k}, i}^{j}\right\|+\left\|u_{n_{k}, i}^{j}-T_{i}^{j} q\right\| \\
& \leq\left\|y_{n_{k}}^{j}-u_{n_{k}, i}^{j}\right\|+d\left(T_{i}^{j} y_{n_{k}}^{j}, T_{i}^{j} q\right) \\
& \leq\left\|y_{n_{k}}^{j}-u_{n_{k}, i}^{j}\right\|+H\left(T_{i}^{j} y_{n_{k}}^{j}, T_{i}^{j} q\right) \\
& \leq\left\|y_{n_{k}}^{j}-u_{n_{k}, i}^{j}\right\|+\left\|y_{n_{k}}^{j}-q\right\|,
\end{aligned}
$$

where $j=1,2, \ldots, k-1$.

Taking lim $\sup _{k \rightarrow \infty}$ of both sides of (30) and from (17) and (27), we have

$$
\begin{aligned}
& \limsup _{k \rightarrow \infty}\left\|x_{n_{k}}-T_{i}^{k} q\right\| \leq \underset{k \rightarrow \infty}{\limsup }\left\|x_{n_{k}}-q\right\|, \\
& \underset{k \rightarrow \infty}{\limsup }\left\|y_{n_{k}}^{j}-T_{i}^{j} q\right\| \leq \underset{k \rightarrow \infty}{\limsup }\left\|y_{n_{k}}^{j}-q\right\| .
\end{aligned}
$$

Now combining (28) with (31) and (29) with (32), we have

$$
\begin{aligned}
& \limsup _{k \rightarrow \infty}\left\|x_{n_{k}}-q\right\|<\limsup _{k \rightarrow \infty}\left\|x_{n_{k}}-q\right\|, \\
& \underset{k \rightarrow \infty}{\limsup }\left\|y_{n_{k}}^{j}-q\right\|<\limsup _{k \rightarrow \infty}\left\|y_{n_{k}}^{j}-q\right\|,
\end{aligned}
$$

which gives contradiction, so we have $T_{i}^{j} q=q$, for $j=$ $1,2, \ldots, k$ and $i \in N$; this implies $q \in F$. Now we prove that $\left\{x_{n}\right\}$ converges weakly to $q$. Let $\left\{x_{m_{k}}\right\}$ be another subsequence of $\left\{x_{n}\right\}$ that converges weakly to some $r \in K$. Again as above 
we conclude that $r \in F$. We show that $q=r$. Let $q \neq r$, since $\lim _{n \rightarrow \infty}\left\|x_{n}-p\right\|$ exists for every $p \in F$. From (1), we have

$$
\begin{aligned}
\lim _{n \rightarrow \infty}\left\|x_{n}-q\right\| & =\limsup _{k \rightarrow \infty}\left\|x_{n_{k}}-q\right\|<\limsup _{k \rightarrow \infty}\left\|x_{n_{k}}-r\right\| \\
& =\limsup _{k \rightarrow \infty}\left\|x_{m_{k}}-r\right\|<\limsup _{k \rightarrow \infty}\left\|x_{m_{k}}-q\right\| \\
& =\lim _{n \rightarrow \infty}\left\|x_{n}-q\right\| .
\end{aligned}
$$

It implies that $\lim _{n \rightarrow \infty}\left\|x_{n}-q\right\|<\lim _{n \rightarrow \infty}\left\|x_{n}-q\right\|$, a contradiction. So we have $q=r$. It means that $\left\{x_{n}\right\}$ converges weakly to $q$ as $n \rightarrow \infty$.

For $T_{i}^{1}=T, T_{i}^{j}=S, j=1,2, \ldots, k-1$, and $T_{i}^{k}=W$, Theorem 7 reduces to the following corollary.

Corollary 8. Let $K$ be a nonempty closed convex subset of a uniformly convex Banach space $X$ with Opial's condition. Let $T$, $S$, and $W$ be three multivalued quasi-nonexpansive mappings from $K$ into $P(K)$ with $F=F(T) \cap F(S) \cap F(W) \neq \varnothing$ and $p \in F$. Let $\left\{x_{n}\right\}$ be the iteration defined as

$$
\begin{gathered}
x_{n+1}=\alpha_{n} x_{n}+\left(1-\alpha_{n}\right) T y_{n}, \\
y_{n}=\beta_{n} x_{n}+\left(1-\beta_{n}\right) S z_{n}, \\
z_{n}=\gamma_{n} x_{n}+\left(1-\gamma_{n}\right) W x_{n},
\end{gathered}
$$

where $y_{n} \in T y_{n}$ such that $d\left(p, y_{n}\right)=d\left(p, T y_{n}\right), z_{n} \in S z_{n}$ such that $d\left(p, z_{n}\right)=d\left(p, S z_{n}\right)$, and $x_{n} \in W x_{n}$ such that $d\left(p, x_{n}\right)=d\left(p, W x_{n}\right),\left\{\alpha_{n}\right\},\left\{\beta_{n}\right\}$, and $\left\{\gamma_{n}\right\}$ are sequences in $[0,1]$ which satisfies $\lim \sup _{n \rightarrow \infty} \alpha_{n}<1$ and $\lim \sup _{n \rightarrow \infty} \beta_{n}<$ $1, \liminf _{n \rightarrow \infty} \alpha_{n}\left(1-\alpha_{n}\right)>0, \liminf _{n \rightarrow \infty} \beta_{n}\left(1-\beta_{n}\right)>0$, and $\liminf _{n \rightarrow \infty} \gamma_{n}\left(1-\gamma_{n}\right)>0$; then $\left\{x_{n}\right\}$ converges weakly to a point $q \in F$.

Theorem 9. Let $X$ be a uniformly convex real Banach space and let $K$ be a bounded and closed convex subset of $X$. For $i \in N$, let $\left\{T_{i}^{j}\right\}_{j=1}^{k}$ be a sequence of multivalued quasinonexpansive and continuous mappings from $K$ into $P(K)$ with $F:=\cap_{i=1}^{\infty} F\left(T_{i}^{j}\right) \neq \varnothing, j=1,2, \ldots, k$ and $p \in F$. Let $\left\{x_{n}\right\}$ be a sequence defined by (2) with $\left\{\alpha_{n, i}\right\}_{i=0}^{\infty}$ and $\left\{\beta_{n, i}^{j}\right\}_{i=0}^{\infty}, j=$ $1,2, \ldots, k-1$ are sequences in $[0,1]$ which satisfies $\sum_{i=0}^{\infty} \alpha_{n, i}=1$ and $\sum_{i=0}^{\infty} \beta_{n, i}^{j}=1, j=1,2, \ldots, k-1, \lim \sup _{n \rightarrow \infty} \alpha_{n, 0}<$ 1 and $\limsup _{n \rightarrow \infty} \beta_{n, 0}^{j}<1, j=1,2, \ldots, k-2$, and $\liminf _{n \rightarrow \infty} \alpha_{n, 0} \alpha_{n, m}>0$ and $\liminf _{n \rightarrow \infty} \beta_{n, 0}^{j} \beta_{n, i}^{j}>0, j=$ $1,2, \ldots, k-1$, for all $i \in N$. Assume that one of $T_{i}^{j}$ is hemicompact. Then $\left\{x_{n}\right\}$ converges strongly to common fixed point of $\left\{T_{i}^{j}\right\}$.

Proof. Let $T_{i}^{j}$ is hemicompact for some $i, j \in N$, then from (17) and (27), we have $\lim _{n \rightarrow \infty} d\left(x_{n}, T_{i}^{k} x_{n}\right)=0$, for all $i \in N$, and $\lim _{n \rightarrow \infty} d\left(y_{n}^{j}, T_{i}^{j} y_{n}^{j}\right)=0$, for all $i \in N$, $j=1,2, \ldots, k-1$. So there exists a subsequence $\left\{x_{n_{k}}\right\}$ of $\left\{x_{n}\right\}$ such that $\lim _{k \rightarrow \infty} x_{n_{k}}=q \in K$; using (25), we can say that $\lim _{k \rightarrow \infty} y_{n_{k}}^{j}=q \in K$. From continuity of
$\left\{T_{i}^{j}\right\}_{j=1}^{k}, i \in N$, we have $\lim _{m \rightarrow \infty} d\left(x_{n_{m}}, T_{i}^{k} x_{n_{m}}\right) \rightarrow d\left(q, T_{i}^{k} q\right)$ and $\lim _{m \rightarrow \infty} d\left(y_{n_{m}}^{j}, T_{i}^{j} y_{n_{m}}^{j}\right) \rightarrow d\left(q, T_{i}^{j} q\right)$. This implies that $d\left(q, T_{i}^{j} q\right)=0, j=1,2, \ldots, k$, and $q \in F$. Since $\lim _{n \rightarrow \infty} \| x_{n}-$ $q \|$ exists, it follows that $\left\{x_{n}\right\}$ converges strongly to $q$.

Theorem 10. Let $X$ be a uniformly convex real Banach space and let $K$ be a compact convex subset of $X$. For $i \in N$, let $\left\{T_{i}^{j}\right\}_{j=1}^{k}$ be a sequence of multivalued quasi-nonexpansive mappings from $K$ into $P(K)$ with $F:=\cap_{i=1}^{\infty} F\left(T_{i}^{j}\right) \neq \varnothing$, $j=1,2, \ldots, k$, and $p \in F$. Let $\left\{x_{n}\right\}$ be a sequence defined by (2) with $\left\{\alpha_{n, i}\right\}_{i=0}^{\infty}$ and $\left\{\beta_{n, i}^{j}\right\}_{i=0}^{\infty}, j=1,2, \ldots, k-1$ are sequences in $[0,1]$ which satisfies $\sum_{i=0}^{\infty} \alpha_{n, i}=1$ and $\sum_{i=0}^{\infty} \beta_{n, i}^{j}=1, j=$ $1,2, \ldots, k-1, \limsup _{n \rightarrow \infty} \alpha_{n, 0}<1$ and $\limsup _{n \rightarrow \infty} \beta_{n, 0}^{j}<$ $1, j=1,2, \ldots, k-2$, and $\liminf _{n \rightarrow \infty} \alpha_{n, 0} \alpha_{n, m}>0$ and $\liminf _{n \rightarrow \infty} \beta_{n, 0}^{j} \beta_{n, i}^{j}>0, j=1,2, \ldots, k-1$, for all $i \in N$. Then $\left\{x_{n}\right\}$ converges strongly to common fixed point of $\left\{T_{i}^{j}\right\}$.

Proof. Since $K$ is compact, there exists a subsequence $\left\{x_{n_{k}}\right\}$ of $\left\{x_{n}\right\}$ such that $\lim _{k \rightarrow \infty}\left\|x_{n_{k}}-q\right\|=0$ for some $q \in K$; also from (25), we can say that

$$
\lim _{k \rightarrow \infty}\left\|y_{n_{k}}^{j}-q\right\|=0, \quad j=1,2, \ldots, k-1
$$

Now, we have

$$
\begin{aligned}
& d\left(q, T_{i}^{k} q\right) \leq d\left(q, x_{n_{k}}\right)+d\left(x_{n_{k}}, T_{i}^{k} x_{n_{k}}\right)+H\left(T_{i}^{k} x_{n_{k}}, T_{i}^{k} q\right) \\
& \leq 2\left\|x_{n_{k}}-q\right\|+d\left(x_{n_{k}}, T_{i}^{k} x_{n_{k}}\right) \longrightarrow 0 \text { as } k \longrightarrow \infty, \\
& d\left(q, T_{i}^{j} q\right) \leq d\left(q, y_{n_{k}}^{j}\right)+d\left(x_{n_{k}}, T_{i}^{j} y_{n_{k}}^{j}\right)+H\left(T_{i}^{j} y_{n_{k}}^{j}, T_{i}^{j} q\right) \\
& \leq 2\left\|y_{n_{k}}^{j}-q\right\|+d\left(y_{n_{k}}^{j}, T_{i}^{j} y_{n_{k}}^{j}\right) \longrightarrow 0 \text { as } k \longrightarrow \infty, \\
& \text { where } j=1,2, \ldots, k-1 .
\end{aligned}
$$

Hence this implies that $q \in F$ and $\left\{x_{n}\right\}$ converges strongly to common fixed point of $\left\{T_{i}^{j}\right\}$.

Remark 11. If in iterative process defined by (6) we use $k=1$ and 2 , Theorems 7,9 , and 10 reduce into convergence results proved by Bunyawat and Suantai $[6,22]$ and Zhang et al. [21].

For $k=3$ and $i=p$ (any finite number), Theorems 7, 9, and 10 reduce into result proved by Ahmed and Altwqi [26].

\subsection{Strong and Weak Convergence Results for New Multistep Iterative Scheme (7)}

Theorem 12. Let $K$ be a nonempty closed convex subset of a uniformly convex Banach space $X$ with Opial's condition. For $i \in N$, let $\left\{T_{i}^{j}\right\}_{j=1}^{k}$ be $k$ sequences of multivalued quasinonexpansive mappings from $K$ into $(K)$ with $F=$ $\cap_{j=1}^{k}\left[\cap_{i=1}^{\infty} T_{i}^{j}\right] \neq \varnothing$ and $p \in F$. Let $\left\{x_{n}\right\}$ be the sequence defined by (7); then it converges weakly to a point $q \in F$. 
Proof. Let $p \in F$, first we prove that $\left\{x_{n}\right\}$ is bounded and $\lim _{n \rightarrow \infty}\left\|x_{n}-p\right\|$ exists. Now, from Lemma 5 and (7), we have

$$
\begin{aligned}
\left\|y_{n}^{k-1}-p\right\|^{2}= & \left\|\beta_{n, 0}^{k-1} x_{n}+\sum_{i=1}^{\infty} \beta_{n, i}^{k-1} u_{n, i}^{k}-p\right\|^{2} \\
\leq & \beta_{n, 0}^{k-1}\left\|x_{n}-p\right\|^{2}+\sum_{i=1}^{\infty} \beta_{n, i}^{k-1}\left\|u_{n, i}^{k}-p\right\|^{2} \\
& -\beta_{n, 0}^{k-1} \beta_{n, m}^{k-1} g\left(\left\|x_{n}-u_{n, m}^{k}\right\|\right) \\
\leq & \beta_{n, 0}^{k-1}\left\|x_{n}-p\right\|^{2}+\sum_{i=1}^{\infty} \beta_{n, i}^{k-1}\left(d\left(u_{n, i}^{k}, T_{i}^{k} p\right)\right)^{2} \\
& -\beta_{n, 0}^{k-1} \beta_{n, m}^{k-1} g\left(\left\|x_{n}-u_{n, m}^{k}\right\|\right) \\
\leq & \beta_{n, 0}^{k-1}\left\|x_{n}-p\right\|^{2}+\sum_{i=1}^{\infty} \beta_{n, i}^{k-1}\left(H\left(T_{i}^{k} x_{n}, T_{i}^{k} p\right)\right)^{2} \\
& -\beta_{n, 0}^{k-1} \beta_{n, m}^{k-1} g\left(\left\|x_{n}-u_{n, m}^{k}\right\|\right) \\
\leq & \beta_{n, 0}^{k-1}\left\|x_{n}-p\right\|^{2}+\sum_{i=1}^{\infty} \beta_{n, i}^{k-1}\left\|x_{n}-p\right\|^{2} \\
& -\beta_{n, 0}^{k-1} \beta_{n, m}^{k-1} g\left(\left\|x_{n}-u_{n, m}^{k}\right\|\right) \\
\leq & \left\|x_{n}-p\right\|^{2}-\beta_{n, 0}^{k-1} \beta_{n, m}^{k-1} g\left(\left\|x_{n}-u_{n, m}^{k}\right\|\right),
\end{aligned}
$$$$
\left\|y_{n}^{k-2}-p\right\|^{2}=\left\|\beta_{n, 0}^{k-2} y_{n}^{k-1}+\sum_{i=1}^{\infty} \beta_{n, i}^{k-2} u_{n, i}^{k-1}-p\right\|^{2}
$$$$
\leq \beta_{n, 0}^{k-2}\left\|y_{n}^{k-1}-p\right\|^{2}+\sum_{i=1}^{\infty} \beta_{n, i}^{k-2}\left\|u_{n, i}^{k-1}-p\right\|^{2}
$$$$
-\beta_{n, 0}^{k-2} \beta_{n, m}^{k-2} \mathcal{g}\left(\left\|y_{n}^{k-1}-u_{n, m}^{k-1}\right\|\right)
$$$$
\leq \beta_{n, 0}^{k-2}\left\|y_{n}^{k-1}-p\right\|^{2}+\sum_{i=1}^{\infty} \beta_{n, i}^{k-2}\left(d\left(u_{n, i}^{k-1}, T_{i}^{k-1} p\right)\right)^{2}
$$$$
-\beta_{n, 0}^{k-2} \beta_{n, m}^{k-2} g\left(\left\|y_{n}^{k-1}-u_{n, m}^{k-1}\right\|\right)
$$$$
\leq \beta_{n, 0}^{k-2}\left\|y_{n}^{k-1}-p\right\|^{2}
$$$$
+\sum_{i=1}^{\infty} \beta_{n, i}^{k-2}\left(H\left(T_{i}^{k-1} y_{n}^{k-1}, T_{i}^{k-1} p\right)\right)^{2}
$$$$
-\beta_{n, 0}^{k-2} \beta_{n, m}^{k-2} g\left(\left\|y_{n}^{k-1}-u_{n, m}^{k-1}\right\|\right)
$$$$
\leq \beta_{n, 0}^{k-2}\left\|y_{n}^{k-1}-p\right\|^{2}+\sum_{i=1}^{\infty} \beta_{n, i}^{k-2}\left\|y_{n}^{k-1}-p\right\|^{2}
$$$$
-\beta_{n, 0}^{k-2} \beta_{n, m}^{k-2} g\left(\left\|y_{n}^{k-1}-u_{n, m}^{k-1}\right\|\right)
$$$$
\leq\left\|y_{n}^{k-1}-p\right\|^{2}-\beta_{n, 0}^{k-2} \beta_{n, m}^{k-2} g\left(\left\|y_{n}^{k-1}-u_{n, m}^{k-1}\right\|\right)
$$$$
\leq\left\|x_{n}-p\right\|^{2}-\beta_{n, 0}^{k-1} \beta_{n, m}^{k-1} g\left(\left\|x_{n}-u_{n, m}^{k}\right\|\right) \text {. }
$$

Similarly, we get

$$
\begin{aligned}
\left\|y_{n}^{k-3}-p\right\|^{2}= & \left\|\beta_{n, 0}^{k-3} y_{n}^{k-2}+\sum_{i=1}^{\infty} \beta_{n, i}^{k-3} u_{n, i}^{k-2}-p\right\|^{2} \\
\leq & \beta_{n, 0}^{k-3}\left\|y_{n}^{k-2}-p\right\|^{2}+\sum_{i=1}^{\infty} \beta_{n, i}^{k-3}\left\|u_{n, i}^{k-2}-p\right\|^{2} \\
& -\beta_{n, 0}^{k-3} \beta_{n, m}^{k-3} g\left(\left\|y_{n}^{k-2}-u_{n, m}^{k-2}\right\|\right) \\
\leq & \beta_{n, 0}^{k-3}\left\|y_{n}^{k-2}-p\right\|^{2}+\sum_{i=1}^{\infty} \beta_{n, i}^{k-3}\left(d\left(u_{n, i}^{k-2}, T_{i}^{k-2} p\right)\right)^{2} \\
& -\beta_{n, 0}^{k-3} \beta_{n, m}^{k-3} g\left(\left\|y_{n}^{k-2}-u_{n, m}^{k-2}\right\|\right) \\
\leq & \beta_{n, 0}^{k-3}\left\|y_{n}^{k-2}-p\right\|^{2} \\
& +\sum_{i=1}^{\infty} \beta_{n, i}^{k-3}\left(H\left(T_{i}^{k-2} y_{n}^{k-2}, T_{i}^{k-2} p\right)\right)^{2} \\
& -\beta_{n, 0}^{k-3} \beta_{n, m}^{k-3} g\left(\left\|y_{n}^{k-2}-u_{n, m}^{k-2}\right\|\right) \\
\leq & \beta_{n, 0}^{k-3}\left\|y_{n}^{k-2}-p\right\|^{2}+\sum_{i=1}^{\infty} \beta_{n, i}^{k-3}\left\|y_{n}^{k-2}-p\right\|^{2} \\
& -\beta_{n, 0}^{k-3} \beta_{n, m}^{k-3} g\left(\left\|y_{n}^{k-2}-u_{n, m}^{k-2}\right\|\right) \\
\leq & \left\|y_{n}^{k-2}-p\right\|^{2}-\beta_{n, 0}^{k-3} \beta_{n, m}^{k-3} g\left(\left\|y_{n}^{k-2}-u_{n, m}^{k-2}\right\|\right),
\end{aligned}
$$

$\left\|y_{n}^{k-3}-p\right\|^{2} \leq\left\|x_{n}-p\right\|^{2}$$$
-\beta_{n, 0}^{k-1} \beta_{n, m}^{k-1} g\left(\left\|x_{n}-u_{n, m}^{k}\right\|\right)
$$$$
-\beta_{n, 0}^{k-3} \beta_{n, m}^{k-3} g\left(\left\|y_{n}^{k-2}-u_{n, m}^{k-2}\right\|\right)
$$$$
\leq\left\|x_{n}-p\right\|^{2}-\beta_{n, 0}^{k-1} \beta_{n, m}^{k-1} g\left(\left\|x_{n}-u_{n, m}^{k}\right\|\right) \text {. }
$$

Now continuing like this at last, we have

$$
\begin{aligned}
\left\|y_{n}^{1}-p\right\| \leq & \left\|x_{n}-p\right\|^{2}-\beta_{n, 0}^{k-1} \beta_{n, m}^{k-1} g\left(\left\|x_{n}-u_{n, m}^{k}\right\|\right) \\
\left\|x_{n+1}-p\right\|^{2} & =\left\|\alpha_{n, 0} y_{n}^{1}+\sum_{i=1}^{\infty} \alpha_{n, i} u_{n, i}^{1}-p\right\|^{2} \\
& \leq \alpha_{n, 0}\left\|y_{n}^{1}-p\right\|^{2}+\sum_{i=1}^{\infty} \alpha_{n, i}\left\|u_{n, i}^{1}-p\right\|^{2} \\
& \leq \alpha_{n, 0}\left\|y_{n}^{1}-p\right\|^{2}+\sum_{i=1}^{\infty} \alpha_{n, i}\left(d\left(u_{n, i}^{1}, T_{i}^{1} p\right)\right)^{2} \\
& \leq \alpha_{n, 0}\left\|y_{n}^{1}-p\right\|^{2}+\sum_{i=1}^{\infty} \alpha_{n, i}\left(H\left(T_{i}^{1} y_{n}^{1}, T_{i}^{1} p\right)\right)^{2} \\
& \leq \alpha_{n, 0}\left\|y_{n}^{1}-p\right\|^{2}+\sum_{i=1}^{\infty} \alpha_{n, i}\left\|y_{n}^{1}-p\right\|^{2} \\
& \leq\left\|y_{n}^{1}-p\right\|^{2} .
\end{aligned}
$$


By putting (41) in (42), we get

$$
\left\|x_{n+1}-p\right\|^{2} \leq\left\|x_{n}-p\right\|^{2}-\beta_{n, 0}^{k-1} \beta_{n, m}^{k-1} g\left(\left\|x_{n}-u_{n, m}^{k}\right\|\right)
$$

So from (43), we say that $\left\{\left\|x_{n}-p\right\|\right\}$ is nondecreasing and bounded and hence, $\left\{x_{n}\right\}$ is bounded and $\lim _{n \rightarrow \infty}\left\|x_{n}-p\right\|$ exists.

Now we prove that $\lim _{n \rightarrow \infty}\left\|x_{n}-u_{n, m}^{k}\right\|=0, \lim _{n \rightarrow \infty} \| x_{n}-$ $y_{n}^{j} \|=0$ and $\lim _{n \rightarrow \infty}\left\|y_{n}^{j}-u_{n, m}^{j}\right\|=0$, where $j=1,2, \ldots, k-1$. From (43), we can write as

$$
\beta_{n, 0}^{k-1} \beta_{n, m}^{k-1} g\left(\left\|x_{n}-u_{n, m}^{k}\right\|\right) \leq\left\|x_{n}-p\right\|^{2}-\left\|x_{n+1}-p\right\|^{2}
$$

Now from (7), as we assume that the following conditions hold: $\limsup _{n \rightarrow \infty} \alpha_{n, 0}<1, \limsup _{n \rightarrow \infty} \beta_{n, 0}^{j}<1, j=$ $1,2, \ldots, k-2, \liminf _{n \rightarrow \infty} \alpha_{n, 0} \alpha_{n, m}>0, \liminf _{n \rightarrow \infty} \beta_{n, 0}^{j} \beta_{n, i}^{j}>$ $0, j=1,2, \ldots, k-1$, and $\lim _{n \rightarrow \infty}\left\|x_{n}-p\right\|$ exist. So from these conditions, we have $\lim _{n \rightarrow \infty} g\left(\left\|x_{n}-u_{n, m}^{k}\right\|\right)=0$ and then using the continuity of $g$, we have

$$
\lim _{n \rightarrow \infty}\left\|x_{n}-u_{n, m}^{k}\right\|=0, \quad \text { for each } m \in N
$$

From (38) and (39), we have

$$
\left\|y_{n}^{k-2}-p\right\|^{2} \leq\left\|x_{n}-p\right\|^{2}-\beta_{n, 0}^{k-2} \beta_{n, m}^{k-2} g\left(\left\|y_{n}^{k-1}-u_{n, m}^{k-1}\right\|\right) .
$$

Now by putting (39) in (40), we have

$$
\left\|y_{n}^{k-3}-p\right\|^{2} \leq\left\|x_{n}-p\right\|^{2}-\beta_{n, 0}^{k-2} \beta_{n, m}^{k-2} g\left(\left\|y_{n}^{k-1}-u_{n, m}^{k-1}\right\|\right) .
$$

Now continuing like this at last, we have

$$
\left\|x_{n+1}-p\right\|^{2} \leq\left\|x_{n}-p\right\|^{2}-\beta_{n, 0}^{k-2} \beta_{n, m}^{k-2} g\left(\left\|y_{n}^{k-1}-u_{n, m}^{k-1}\right\|\right)
$$

we can write it as

$$
\beta_{n, 0}^{k-2} \beta_{n, m}^{k-2} g\left(\left\|y_{n}^{k-1}-u_{n, m}^{k-1}\right\|\right) \leq\left\|x_{n}-p\right\|^{2}-\left\|x_{n+1}-p\right\|^{2} .
$$

Since we assume that $\liminf _{n \rightarrow \infty} \beta_{n, 0}^{j} \beta_{n, i}^{j}>0, j=1,2, \ldots, k-$ 1 and $\lim _{n \rightarrow \infty}\left\|x_{n}-p\right\|$ exists, we have $\lim _{n \rightarrow \infty} g\left(\| y_{n}^{k-1}-\right.$ $\left.u_{n, m}^{k-1} \|\right)=0$ and from the continuity of $g$, we have

$$
\lim _{n \rightarrow \infty}\left\|y_{n}^{k-1}-u_{n, m}^{k-1}\right\|=0, \quad \text { for each } m \in N
$$

So by repeating these steps for different values of $j=$ $1,2, \ldots, k-2$, we have

$$
\begin{array}{r}
\lim _{n \rightarrow \infty}\left\|y_{n}^{j}-u_{n, m}^{j}\right\|=0, \quad \text { for each } m \in N, \\
\text { where } j=1,2, \ldots, k-1 .
\end{array}
$$

Now, we prove that $\lim _{n \rightarrow \infty}\left\|x_{n}-y_{n}^{j}\right\|=0$, from (7), we have

$$
\begin{aligned}
\left\|x_{n}-y_{n}^{k-1}\right\| & =\left\|x_{n}-\beta_{n, 0}^{k-1} x_{n}-\sum_{i=1}^{\infty} \beta_{n, i}^{k-1} u_{n, i}^{k}\right\|, \\
& \text { where } u_{n, i}^{k} \in T_{i}^{k} x_{n}, \\
\left\|x_{n}-y_{n}^{k-1}\right\| \leq \beta_{n, 0}^{k-1}\left\|x_{n}-x_{n}\right\|+\sum_{i=1}^{\infty} \beta_{n, i}^{k-1}\left\|x_{n}-u_{n, i}^{k}\right\| & \\
\leq & \sum_{i=1}^{\infty} \beta_{n, i}^{k-1}\left\|x_{n}-u_{n, i}^{k}\right\| .
\end{aligned}
$$

Taking limit $n \rightarrow \infty$ of both sides of (53) and using (45), we have

$$
\lim _{n \rightarrow \infty}\left\|x_{n}-y_{n}^{k-1}\right\|=0
$$

Again using (7), we have

$$
\begin{aligned}
\left\|x_{n}-y_{n}^{k-2}\right\| & =\left\|x_{n}-\beta_{n, 0}^{k-2} y_{n}^{k-1}-\sum_{i=1}^{\infty} \beta_{n, i}^{k-2} u_{n, i}^{k-1}\right\| \\
& \leq \beta_{n, 0}^{k-2}\left\|x_{n}-y_{n}^{k-1}\right\|+\beta_{n, i}^{k-2}\left\|x_{n}-u_{n, i}^{k-1}\right\|, \\
\left\|x_{n}-u_{n, i}^{k-1}\right\| & \leq\left\|x_{n}-y_{n}^{k-1}\right\|+\left\|y_{n}^{k-1}-u_{n, i}^{k-1}\right\| .
\end{aligned}
$$

From (51) and (54), we have $\lim _{n \rightarrow \infty}\left\|x_{n}-y_{n}^{k-2}\right\|=0$. Now by repeating these steps for all values of $j=1,2, \ldots, k-3$, we get

$$
\lim _{n \rightarrow \infty}\left\|x_{n}-y_{n}^{j}\right\|=0, \quad \text { where } j=1,2, \ldots, k-1
$$

Now to prove weak convergence of $\left\{x_{n}\right\}$ to a point $q \in F$, we will use (43); that is, $\left\{x_{n}\right\}$ is bounded, so there exists a subsequence $\left\{x_{n_{k}}\right\}_{k=1}^{\infty}$ of $\left\{x_{n}\right\}$ such that $x_{n_{k}}$ converges weakly to $q \in K$; then using (56), we claim that $y_{n_{k}}^{j}$ converges weakly to $q \in K$, for $j=1,2, \ldots, k-1$. Now assume that there exist $i \in N$, such that $T_{i}^{j} q \neq q$, for $j=1,2, \ldots, k$; then by Opial's condition we have

$$
\begin{array}{r}
\limsup _{n \rightarrow \infty}\left\|x_{n_{k}}-q\right\|<\limsup _{n \rightarrow \infty}\left\|x_{n_{k}}-T_{i}^{k} q\right\|, \\
\limsup _{n \rightarrow \infty}\left\|y_{n_{k}}^{j}-q\right\|<\limsup _{n \rightarrow \infty}\left\|y_{n_{k}}^{j}-T_{i}^{j} q\right\|, \\
\text { where } j=1,2, \ldots, k-1 .
\end{array}
$$


As $\left\{T_{i}^{j}\right\}_{j=1}^{k}$ are $k$-multivalued quasi nonexpansive mappings, we have

$$
\begin{aligned}
\left\|x_{n_{k}}-T_{i}^{k} q\right\| & \leq\left\|x_{n_{k}}-u_{n_{k}, i}^{k}\right\|+\left\|u_{n_{k}, i}^{k}-T_{i}^{k} q\right\| \\
& \leq\left\|x_{n_{k}}-u_{n_{k}, i}^{k}\right\|+d\left(T_{i}^{k} x_{n_{k}}, T_{i}^{k} q\right) \\
& \leq\left\|x_{n_{k}}-u_{n_{k}, i}^{k}\right\|+H\left(T_{i}^{k} x_{n_{k}}, T_{i}^{k} q\right) \\
& \leq\left\|x_{n_{k}}-u_{n_{k}, i}^{k}\right\|+\left\|x_{n_{k}}-q\right\|, \\
\left\|y_{n_{k}}^{j}-T_{i}^{j} q\right\| & \leq\left\|y_{n_{k}}^{j}-u_{n_{k}, i}^{j}\right\|+\left\|u_{n_{k}, i}^{j}-T_{i}^{j} q\right\| \\
& \leq\left\|y_{n_{k}}^{j}-u_{n_{k}, i}^{j}\right\|+d\left(T_{i}^{j} y_{n_{k}}^{j}, T_{i}^{j} q\right) \\
& \leq\left\|y_{n_{k}}^{j}-u_{n_{k}, i}^{j}\right\|+H\left(T_{i}^{j} y_{n_{k}}^{j}, T_{i}^{j} q\right) \\
& \leq\left\|y_{n_{k}}^{j}-u_{n_{k}, i}^{j}\right\|+\left\|y_{n_{k}}^{j}-q\right\|,
\end{aligned}
$$

where $j=1,2, \ldots, k-1$.

Taking lim $\sup _{k \rightarrow \infty}$ of both sides of (59) and from (45) and (51), we have

$$
\begin{aligned}
& \limsup _{k \rightarrow \infty}\left\|x_{n_{k}}-T_{i}^{k} q\right\| \leq \underset{k \rightarrow \infty}{\limsup }\left\|x_{n_{k}}-q\right\|, \\
& \limsup _{k \rightarrow \infty}\left\|y_{n_{k}}^{j}-T_{i}^{j} q\right\| \leq \underset{k \rightarrow \infty}{\limsup }\left\|y_{n_{k}}^{j}-q\right\| .
\end{aligned}
$$

Now combining (57) with (60) and (58) with (61), we have

$$
\begin{gathered}
\limsup _{k \rightarrow \infty}\left\|x_{n_{k}}-q\right\|<\limsup _{k \rightarrow \infty}\left\|x_{n_{k}}-q\right\|, \\
\limsup _{k \rightarrow \infty}\left\|y_{n_{k}}^{j}-q\right\|<\limsup _{k \rightarrow \infty}\left\|y_{n_{k}}^{j}-q\right\|,
\end{gathered}
$$

which gives contradiction, so we have $T_{i}^{j} q=q$, for $j=$ $1,2, \ldots, k$ and $i \in N$; this implies that $q \in F$. Now we prove that $\left\{x_{n}\right\}$ converges weakly to $q$. Let $\left\{x_{m_{k}}\right\}$ be another subsequence of $\left\{x_{n}\right\}$ that converges weakly to some $r \in K$. Again as above we conclude that $r \in F$. We show that $q=r$. Let $q \neq r$, since $\lim _{n \rightarrow \infty}\left\|x_{n}-p\right\|$ exists for every $p \in F$. From (1), we have

$$
\begin{aligned}
\lim _{n \rightarrow \infty}\left\|x_{n}-q\right\| & =\limsup _{k \rightarrow \infty}\left\|x_{n_{k}}-q\right\|<\limsup _{k \rightarrow \infty}\left\|x_{n_{k}}-r\right\| \\
& =\limsup _{k \rightarrow \infty}\left\|x_{m_{k}}-r\right\|<\limsup _{k \rightarrow \infty}\left\|x_{m_{k}}-q\right\| \\
& =\lim _{n \rightarrow \infty}\left\|x_{n}-q\right\| .
\end{aligned}
$$

It implies that $\lim _{n \rightarrow \infty}\left\|x_{n}-q\right\|<\lim _{n \rightarrow \infty}\left\|x_{n}-q\right\|$, a contradiction. So we have $q=r$. It means that $\left\{x_{n}\right\}$ converges weakly to $q$ as $n \rightarrow \infty$.

Theorem 13. Let $X$ be a uniformly convex real Banach space and let $K$ be a bounded and closed convex subset of $X$. For $i \in N$, let $\left\{T_{i}^{j}\right\}_{j=1}^{k}$ be a sequence of multivalued quasinonexpansive and continuous mappings from $K$ into $P(K)$ with
$F:=\cap_{i=1}^{\infty} F\left(T_{i}^{j}\right) \neq \varnothing, j=1,2, \ldots, k$ and $p \in F$. Let $\left\{x_{n}\right\}$ be a sequence defined by (7) with $\left\{\alpha_{n, i}\right\}_{i=0}^{\infty}$ and $\left\{\beta_{n, i}^{j}\right\}_{i=0}^{\infty}, j=$ $1,2, \ldots, k-1$ are sequences in $[0,1]$ which satisfies $\sum_{i=0}^{\infty} \alpha_{n, i}=1$ and $\sum_{i=0}^{\infty} \beta_{n, i}^{j}=1, j=1,2, \ldots, k-1, \liminf _{n \rightarrow \infty} \beta_{n, 0}^{j} \beta_{n, i}^{j}>0$, $j=1,2, \ldots, k-1$, for all $i \in N$. Assume that one of $T_{i}^{j}$ is hemicompact. Then $\left\{x_{n}\right\}$ converges strongly to common fixed point of $\left\{T_{i}^{j}\right\}$.

Proof. Let $T_{i}^{j}$ is hemicompact for some $i, j \in N$; then from (45) and (50), we have $\lim _{n \rightarrow \infty} d\left(x_{n}, T_{i}^{k} x_{n}\right)=0$, for all $i \in N$, and $\lim _{n \rightarrow \infty} d\left(y_{n}^{j}, T_{i}^{j} y_{n}^{j}\right)=0$, for all $i \in N$, $j=1,2, \ldots, k-1$. So there exists a subsequence $\left\{x_{n_{k}}\right\}$ of $\left\{x_{n}\right\}$ such that $\lim _{k \rightarrow \infty} x_{n_{k}}=q \in K$ using (56); we can say that $\lim _{k \rightarrow \infty} y_{n_{k}}^{j}=q \in K$. From continuity of $\left\{T_{i}^{j}\right\}_{j=1}^{k}, i \in N$, we have $\lim _{m \rightarrow \infty} d\left(x_{n_{m}}, T_{i}^{k} x_{n_{m}}\right) \rightarrow d\left(q, T_{i}^{k} q\right)$ and $\lim _{m \rightarrow \infty} d\left(y_{n_{m}}^{j}, T_{i}^{j} y_{n_{m}}^{j}\right) \rightarrow d\left(q, T_{i}^{j} q\right)$. This implies that $d\left(q, T_{i}^{j} q\right)=0, j=1,2, \ldots, k$ and $q \in F$. Since $\lim _{n \rightarrow \infty}\left\|x_{n}-q\right\|$ exists, it follows that $\left\{x_{n}\right\}$ converges strongly to $q$.

Theorem 14. Let $X$ be a uniformly convex real Banach space and let $K$ be a compact convex subset of $X$. For $i \in N$, let $\left\{T_{i}^{j}\right\}_{j=1}^{k}$ be a sequence of multivalued quasi-nonexpansive mappings from $K$ into $P(K)$ with $F:=\cap_{i=1}^{\infty} F\left(T_{i}^{j}\right) \neq \varnothing, j=1,2, \ldots, k$ and $p \in F$. Let $\left\{x_{n}\right\}$ be a sequence defined by (7) with $\left\{\alpha_{n, i}\right\}_{i=0}^{\infty}$ and $\left\{\beta_{n, i}^{j}\right\}_{i=0}^{\infty}, j=1,2, \ldots, k-1$ are sequences in $[0,1]$ which satisfies $\sum_{i=0}^{\infty} \alpha_{n, i}=1$ and $\sum_{i=0}^{\infty} \beta_{n, i}^{j}=1, j=1,2, \ldots, k-1$, $\liminf _{n \rightarrow \infty} \beta_{n, 0}^{j} \beta_{n, i}^{j}>0, j=1,2, \ldots, k-1$, for all $i \in N$. Then $\left\{x_{n}\right\}$ converges strongly to common fixed point of $\left\{T_{i}^{j}\right\}$.

Proof. Since $K$ is compact, there exists a subsequence $\left\{x_{n_{k}}\right\}$ of $\left\{x_{n}\right\}$, such that $\lim _{k \rightarrow \infty}\left\|x_{n_{k}}-q\right\|=0$ for some $q \in K$; also from (56), we can say that

$$
\lim _{k \rightarrow \infty}\left\|y_{n_{k}}^{j}-q\right\|=0, \quad j=1,2, \ldots, k-1 .
$$

Now, we have

$$
\begin{aligned}
& d\left(q, T_{i}^{k} q\right) \leq d\left(q, x_{n_{k}}\right)+d\left(x_{n_{k}}, T_{i}^{k} x_{n_{k}}\right)+H\left(T_{i}^{k} x_{n_{k}}, T_{i}^{k} q\right) \\
& \leq 2\left\|x_{n_{k}}-q\right\|+d\left(x_{n_{k}}, T_{i}^{k} x_{n_{k}}\right) \longrightarrow 0 \text { as } k \longrightarrow \infty, \\
& d\left(q, T_{i}^{j} q\right) \leq d\left(q, y_{n_{k}}^{j}\right)+d\left(x_{n_{k}}, T_{i}^{j} y_{n_{k}}^{j}\right)+H\left(T_{i}^{j} y_{n_{k}}^{j}, T_{i}^{j} q\right) \\
& \leq 2\left\|y_{n_{k}}^{j}-q\right\|+d\left(y_{n_{k}}^{j}, T_{i}^{j} y_{n_{k}}^{j}\right) \longrightarrow 0 \text { as } k \longrightarrow \infty, \\
& \text { where } j=1,2, \ldots, k-1 .
\end{aligned}
$$

Hence this implies that $q \in F$ and $\left\{x_{n}\right\}$ converges strongly to common fixed point of $\left\{T_{i}^{j}\right\}$. 
TABLE 1: Showing the convergence of different iterative sequences to common fixed point $\{0\}$ of countable family of multivalued quasi nonexpansive mappings. From the table we can compare the converging step of one-step, two-step, multistep (6), and multistep (7) iterative schemes and conclude that multistep iterative scheme (7) converges faster than one-step, two-step, and multistep iterative schemes (6).

\begin{tabular}{|c|c|c|c|c|c|c|}
\hline \multirow{2}{*}{$N$} & \multicolumn{4}{|c|}{ Multistep iterative scheme (6) } & \multicolumn{2}{|c|}{ Multistep iterative scheme (7) } \\
\hline & 1-step & 2-step & 3-step & 5-step & 3-step & 5-step \\
\hline 1 & 0.104167 & $8.09766 e-007$ & $5.00012 e-008$ & $5.00001 e-008$ & $1.28445 e-012$ & $3.09053 e-024$ \\
\hline 2 & 0.0425347 & $1.31144 e-012$ & $5.00024 e-015$ & $5.00002 e-015$ & $3.29962 e-024$ & $1.91028 e-047$ \\
\hline 3 & 0.0173683 & $2.12392 e-018$ & $5.00036 e-022$ & $5.00002 e-022$ & $8.47637 e-036$ & $1.18075 e-070$ \\
\hline 11 & $1.34239 e-005$ & $1.0052 e-064$ & $5.00133 e-078$ & $5.00008 e-078$ & $1.60762 e-128$ & $2.51572 e-256$ \\
\hline 12 & $5.48141 e-006$ & $1.62795 e-070$ & $5.00145 e-085$ & $5.00009 e-085$ & $4.12981 e-140$ & $1.55498 e-279$ \\
\hline 13 & $2.23824 e-006$ & $2.63652 e-076$ & $5.00157 e-092$ & $5.0001 e-092$ & $1.06091 e-151$ & $9.61145 e-303$ \\
\hline 14 & $9.13948 e-007$ & $4.26993 e-082$ & $5.00169 e-099$ & $5.00011 e-099$ & $2.72536 e-163$ & $\mathbf{0}$ \\
\hline 15 & $3.73196 e-007$ & $6.91528 e-088$ & $5.00181 e-106$ & $5.00011 e-106$ & $7.00116 e-175$ & $\mathbf{0}$ \\
\hline 16 & $1.52388 e-007$ & $1.11995 e-093$ & $5.00193 e-113$ & $5.00012 e-113$ & $1.79852 e-186$ & $\mathbf{0}$ \\
\hline 27 & $8.01889 e-012$ & $2.25155 e-157$ & $5.00325 e-190$ & $5.00021 e-190$ & $5.78269 e-314$ & $\mathbf{0}$ \\
\hline 28 & $3.27438 e-012$ & $3.64645 e-163$ & $5.00338 e-197$ & $5.00021 e-197$ & $\mathbf{0}$ & $\mathbf{0}$ \\
\hline 29 & $1.33704 e-012$ & $5.90554 e-169$ & $5.0035 e-204$ & $5.00022 e-204$ & $\mathbf{0}$ & $\mathbf{0}$ \\
\hline 46 & $3.26134 e-019$ & $2.14229 e-267$ & $4.94066 e-323$ & $4.94066 e-323$ & $\mathbf{0}$ & $\mathbf{0}$ \\
\hline 47 & $1.33171 e-019$ & $3.4695 e-273$ & 0 & o & $\mathbf{0}$ & $\mathbf{0}$ \\
\hline 48 & $5.43783 e-020$ & $5.61897 e-279$ & $\mathbf{0}$ & $\mathbf{0}$ & $\mathbf{0}$ & $\mathbf{0}$ \\
\hline 55 & $1.02927 e-022$ & $8.79091 e-320$ & $\mathbf{0}$ & $\mathbf{0}$ & $\mathbf{0}$ & $\mathbf{0}$ \\
\hline 56 & $4.20286 e-023$ & 0 & $\mathbf{0}$ & $\mathbf{0}$ & $\mathbf{0}$ & $\mathbf{0}$ \\
\hline 57 & $1.71617 e-023$ & 0 & $\mathbf{0}$ & $\mathbf{0}$ & $\mathbf{0}$ & $\mathbf{0}$ \\
\hline 829 & $4.94066 e-324$ & 0 & $\mathbf{0}$ & $\mathbf{0}$ & $\mathbf{0}$ & $\mathbf{0}$ \\
\hline 830 & 0 & 0 & $\mathbf{0}$ & $\mathbf{0}$ & $\mathbf{0}$ & $\mathbf{0}$ \\
\hline 831 & $\mathbf{0}$ & $\mathbf{0}$ & $\mathbf{0}$ & $\mathbf{0}$ & $\mathbf{0}$ & $\mathbf{0}$ \\
\hline
\end{tabular}

Remark 15. Since iterations used in $[4-6,15,21]$ are special case of iterative scheme (6), motivated from them, we generalize in the following sense.

(1) Since we prove our result for quasi-nonexpansive mappings so generalizes from nonexpansive to quasi nonexpansive.

(2) We generalize from single-valued to multivalued mappings.

(3) Our results extended from one and two countable families to $k$-number of countable families of multivalued quasi-nonexpansive mappings.

(4) We prove weak and strong convergence results for new multistep iterative scheme (7). With the help of numerical example of multivalued quasinonexpansive mappings and computational program in $\mathrm{C}++$ we prove fast rate of convergence of new multistep iterative scheme (7).

\section{Numerical Computation}

We use the following numerical example of finite family of multivalued quasi-nonexpansive mappings to compare the converging steps of one-step, two-step, and two new multistep iterative procedures. Let $\left\{T_{i}^{j}\right\}:[0,1] \rightarrow[0,1]$ be $k$-countable family of multivalued quasi-nonexpansive mappings defined as

$T_{i}^{j} x=\left[0, \frac{x}{i+j+1}\right]$, where $x \in[0,1], i \in N, j=1,2, \ldots, k$.

Now using the initial value $x_{0}=0.5$ and different initial conditions used in result proved above in $\mathrm{C}++$ program, we get the following observation for different iterations.

\section{Conclusion}

After analyzing the comparison shown in Table 1 we conclude that the iterative scheme (7) converges faster than other existing iterative schemes and rate of convergence increases as the number of step of iterations increases for multivalued quasi-nonexpansive mappings.

\section{Conflict of Interests}

The authors declare that there is no conflict of interests regarding the publication of this paper.

\section{References}

[1] S. B. Nadler Jr., "Multi-valued contraction mappings," Pacific Journal of Mathematics, vol. 30, pp. 475-488, 1969. 
[2] J. T. Markin, "Continuous dependence of fixed point sets," Proceedings of the American Mathematical Society, vol. 38, pp. 545-547, 1973.

[3] T. Hu, J.-C. Huang, and B. E. Rhoades, "A general principle for Ishikawa iterations for multi-valued mappings," Indian Journal of Pure and Applied Mathematics, vol. 28, no. 8, pp. 1091-1098, 1997.

[4] K. P. Sastry and G. V. Babu, "Convergence of Ishikawa iterates for a multi-valued mapping with a fixed point," Czechoslovak Mathematical Journal, vol. 55, no. 4, pp. 817-826, 2005.

[5] M. Abbas, S. H. Khan, A. R. Khan, and R. P. Agarwal, "Common fixed points of two multivalued nonexpansive mappings by onestep iterative scheme," Applied Mathematics Letters, vol. 24, no. 2, pp. 97-102, 2011.

[6] A. Bunyawat and S. Suantai, "Convergence theorems for infinite family of multivalued quasi-nonexpansive mappings in uniformly convex Banach spaces," Abstract and Applied Analysis, vol. 2012, Article ID 435790, 6 pages, 2012.

[7] P. Sunthrayuth and P. Kumam, "A general iterative algorithm for the solution of variational inequalities for a nonexpansive semigroup in Banach spaces," Journal of Nonlinear Analysis and Optimization, vol. 1, no. 1, pp. 139-150, 2010.

[8] P. Sunthrayuth and P. Kumam, "Iterative methods for variational inequality problems and fixed point problems of a countable family of strict pseudo-contractions in a q-uniformly smooth Banach space," Fixed Point Theory and Applications, vol. 2012, article 65, 2012.

[9] P. Sunthrayuth and P. Kumam, "Viscosity approximation methods based on generalized contraction mappings for a countable family of strict pseudo-contractions, a general system of variational inequalities and a generalized mixed equilibrium problem in Banach spaces," Mathematical and Computer Modelling, vol. 58, no. 11-12, pp. 1814-1828, 2013.

[10] P. Katchang and P. Kumam, "Strong convergence of the modified Ishikawa iterative method for infinitely many nonexpansive mappings in Banach spaces," Computers and Mathematics with Applications, vol. 59, no. 4, pp. 1473-1483, 2010.

[11] P. Sunthrayuth and P. Kumam, "Strong convergence theorems of a general iterative process for two nonexpansive mappings in Banach spaces," Journal of Computational Analysis and Applications, vol. 14, no. 3, pp. 446-457, 2012.

[12] P. Sunthrayuth and P. Kumam, "A new composite general iterative scheme for nonexpansive semigroups in Banach spaces," International Journal of Mathematics and Mathematical Sciences, vol. 2011, Article ID 560671, 18 pages, 2011.

[13] P. Phuangphoo and P. Kumam, "An iterative procedure for solving the common solution of two total quasi- $\phi$-asymptotically nonexpansive multi-valued mappings in Banach spaces," Journal of Applied Mathematics and Computing, vol. 42, no. 1-2, pp. 321-338, 2013.

[14] L. Górniewicz, Topological Fixed Point Theory of Multivalued Mappings, vol. 495 of Mathematics and Its Applications, Kluwer Academic Publishers, Dordrecht, The Netherlands, 1999.

[15] M. Abbas and B. E. Rhoades, "Fixed point theorems for two new classes of multivalued mappings," Applied Mathematics Letters, vol. 22, no. 9, pp. 1364-1368, 2009.

[16] N. Shahzad and H. Zegeye, "On Mann and Ishikawa iteration schemes for multi-valued maps in Banach spaces," Nonlinear Analysis: Theory, Methods \& Applications, vol. 71, no. 3-4, pp. 838-844, 2009.
[17] W. Cholamjiak and S. Suantai, "A hybrid method for a countable family of multivalued maps, equilibrium problems, and variational inequality problems," Discrete Dynamics in Nature and Society, vol. 2010, Article ID 349158, 14 pages, 2010.

[18] S. Hong, "Fixed points of multivalued operators in ordered metric spaces with applications," Nonlinear Analysis: Theory, Methods \& Applications, vol. 72, no. 11, pp. 3929-3942, 2010.

[19] S. H. Khan, I. Yildirim, and B. E. Rhoades, "A one-step iterative process for two multivalued nonexpansive mappings in Banach spaces," Computers and Mathematics with Applications, vol. 61, no. 10, pp. 3172-3178, 2011.

[20] S.-S. Chang, J. K. Kim, and X. R. Wang, "Modified block iterative algorithm for solving convex feasibility problems in Banach spaces," Journal of Inequalities and Applications, vol. 2010, Article ID 869684, 14 pages, 2010.

[21] F. Zhang, H. Zhang, and Y. Zhang, "New iterative algorithm for two infinite families of multivalued quasi-nonexpansive mappings in uniformly convex Banach spaces," Journal of Applied Mathematics, vol. 2013, Article ID 649537, 7 pages, 2013.

[22] A. Bunyawat and S. Suantai, "Common fixed points of a countable family of multivalued quasi-nonexpansive mappings in uniformly convex Banach spaces," International Journal of Computer Mathematics, vol. 89, no. 16, pp. 2274-2279, 2012.

[23] J. Schu, "Weak and strong convergence to fixed points of asymptotically nonexpansive mappings," Bulletin of the Australian Mathematical Society, vol. 43, no. 1, pp. 153-159, 1991.

[24] L. S. Liu, "Ishikawa and Mann iterative process with errors for nonlinear strongly accretive mappings in Banach spaces," Journal of Mathematical Analysis and Applications, vol. 194, no. 1, pp. 114-125, 1995.

[25] H.-K. Xu, "Iterative algorithms for nonlinear operators," Journal of the London Mathematical Society, vol. 66, no. 1, pp. 240-256, 2002.

[26] R. Ahmed and S. Altwqi, "Convergence theorems for three finite families of multivalued nonexpansive mappings," Journal of the Egyptian Mathematical Society, vol. 22, no. 3, pp. 459-465, 2014. 


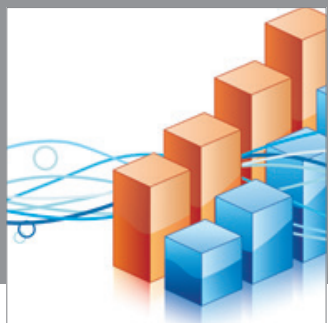

Advances in

Operations Research

mansans

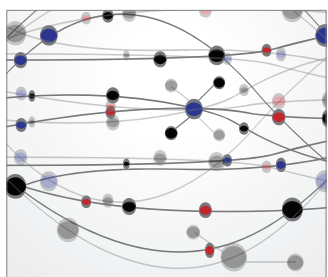

The Scientific World Journal
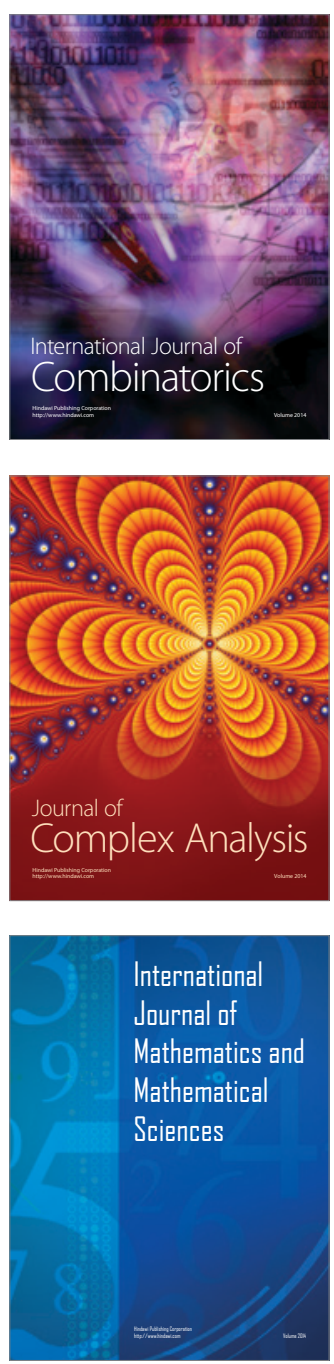
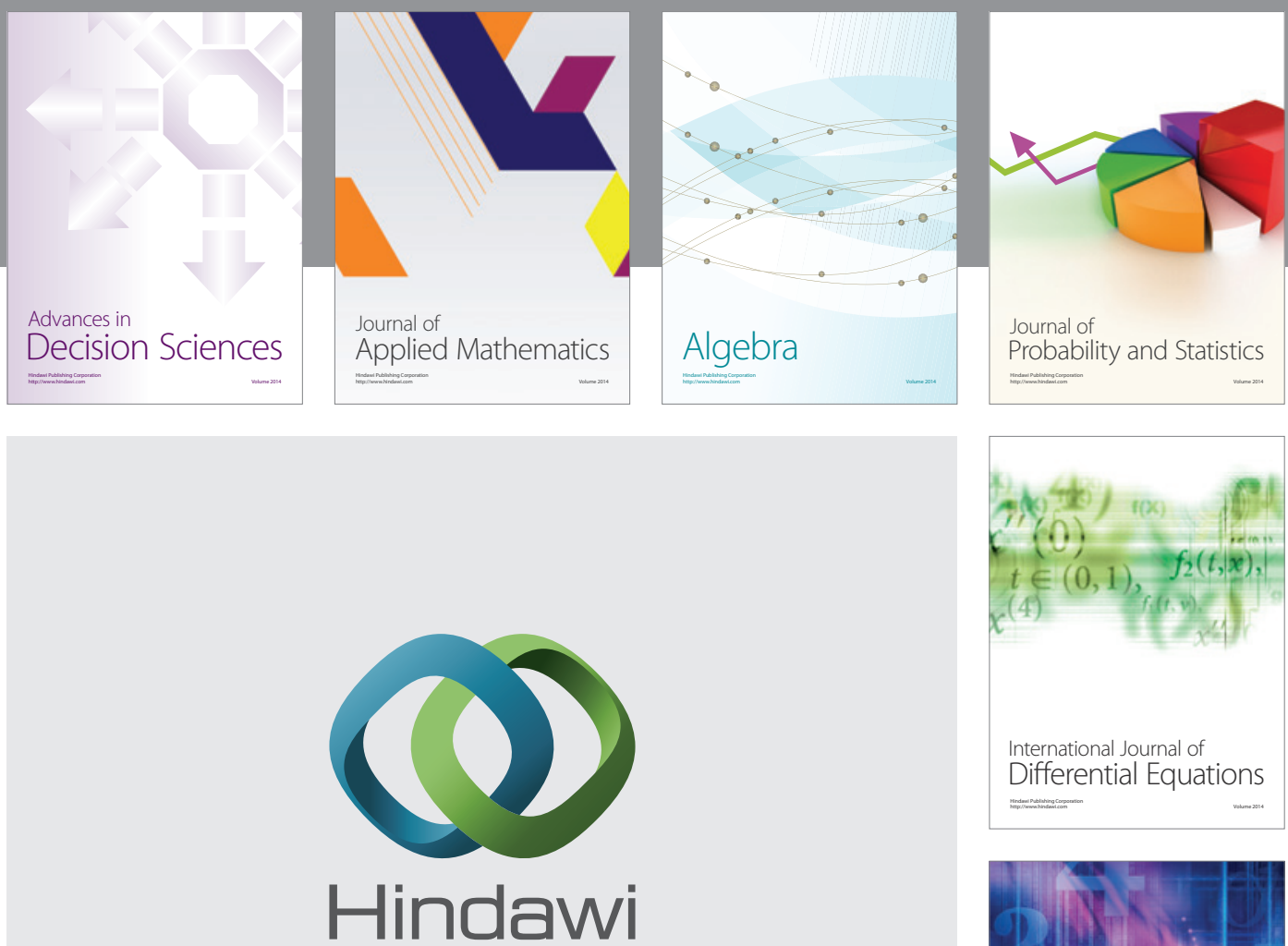

Submit your manuscripts at http://www.hindawi.com
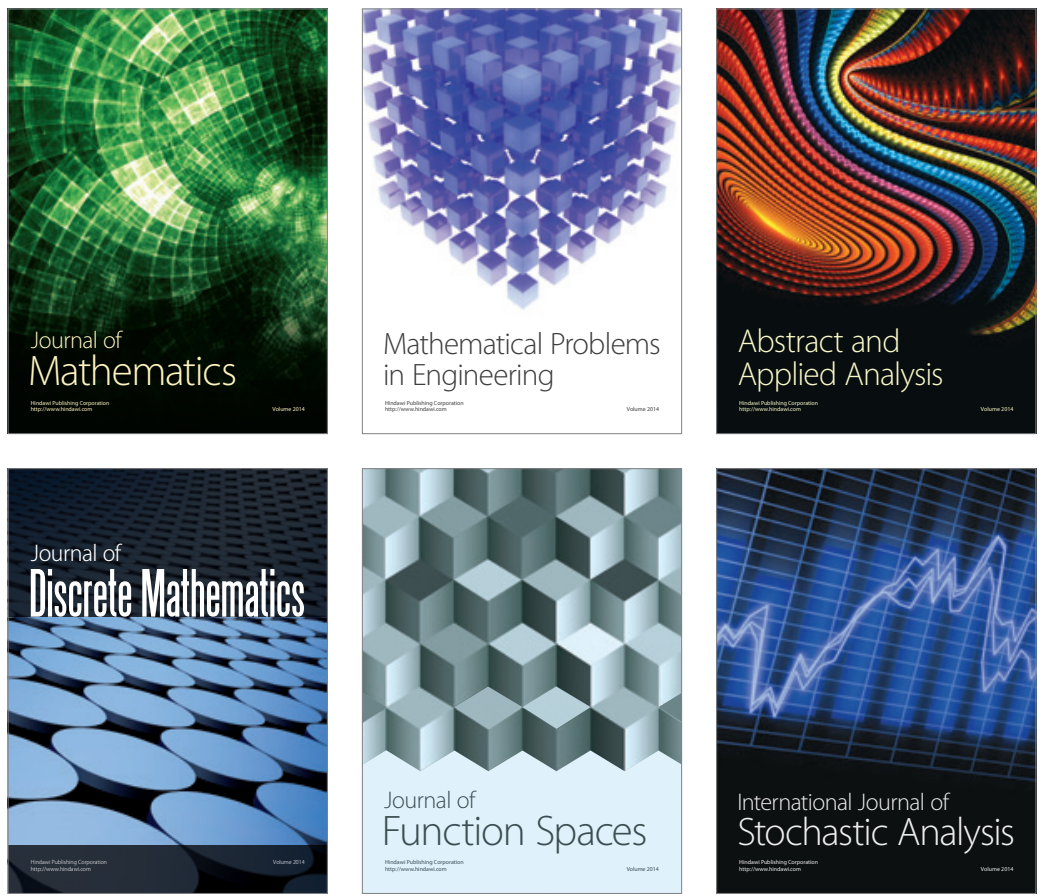

Journal of

Function Spaces

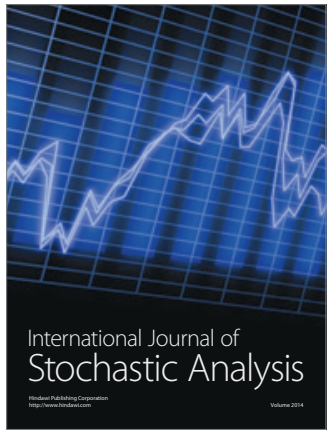

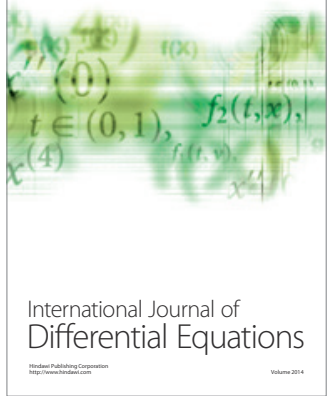
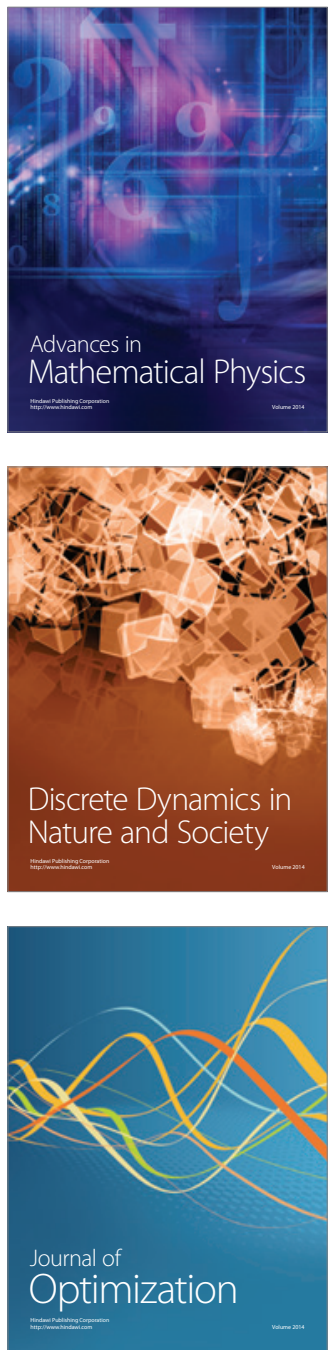